\title{
Fifty years of Shannon information theory in assessing the accuracy and agreement of diagnostic tests
}

\author{
Alberto Casagrande ${ }^{1} \cdot$ Francesco Fabris $^{1}$ (D) $\cdot$ Rossano Girometti $^{2}$
}

Received: 10 June 2021 / Accepted: 17 December 2021 / Published online: 23 February 2022

(C) The Author(s) 2022

\begin{abstract}
Since 1948, Shannon theoretic methods for modeling information have found a wide range of applications in several areas where information plays a key role, which goes well beyond the original scopes for which they have been conceived, namely data compression and error correction over a noisy channel. Among other uses, these methods have been applied in the broad field of medical diagnostics since the 1970s, to quantify diagnostic information, to evaluate diagnostic test performance, but also to be used as technical tools in image processing and registration. This review illustrates the main contributions in assessing the accuracy of diagnostic tests and the agreement between raters, focusing on diagnostic test performance measurements and paired agreement evaluation. This work also presents a recent unified, coherent, and hopefully, final information-theoretical approach to deal with the flows of information involved among the patient, the diagnostic test performed to appraise the state of disease, and the raters who are checking the test results. The approach is assessed by considering two case studies: the first one is related to evaluating extra-prostatic cancers; the second concerns the quality of rapid tests for COVID-19 detection.
\end{abstract}

Keywords Diagnostic information · Diagnostic test performance · Quality measures · Inter-rater agreement · Shannon information theory $\cdot$ Informational diagnostic channels

\section{Introduction}

Claude Shannon's "A mathematical theory of communication" [67], published in 1948, is the milestone paper of the new information age, flourished in the second half of 1900s, which is characterized by a finely branched network that connects each computer, smartphone, terminal, or device we use in our daily life. Shannon's work, which describes

Francesco Fabris

ffabris@units.it

Alberto Casagrande

acasagrande@units.it

Rossano Girometti

rossano.girometti@uniud.it

1 Dipartimento di Matematica e Geoscienze, Università degli Studi di Trieste, Trieste, Italy

2 Istituto di Radiologia, Dipartimento di Area Medica, Università degli Studi di Udine, Ospedale S. Maria della Misericordia, Udine, Italy the fundamental laws of data compression and error correction over a noisy channel, marks the birth of a unifying theory, i.e., Information Theory (IT), with profound intersections with probability, statistics, computer science, and many other fields [75]. Shannon's paper also introduced some novel concepts which aimed to measure the quantity of information either stored in a string or transferred through a communication process: they are, respectively, (Shannon) entropy, related to the data compression problem, and channel capacity, which models the flow of information over a noisy channel, and is defined in terms of the Mutual Information $(M I)$ between input and output.

Communication theory was the first discipline to adopt these information measures, but several attempts were made to export these concepts also in many other fields, such as statistical mechanics [38], statistical inference [48, 81], linguistics [76], taxonomy [47], psychology [8], molecular dynamics [34], computational biology [17], molecular biology [27], genomics [44], neurobiology [26], pattern recognition [49], machine learning [37], deep learning [40], computer vision [66], perception [22], image processing [30], and many others. In some cases, however, these measures have been used in an uncritical way and outside 
a proper theory-safe environment, so that only questionable and partial results were produced. In other cases, when the problems of these external disciplines have been interpreted in agreement with the $I T$ spirit, an informational-theoretic approach to the discipline was set.

This also occurred in medical diagnostics, where clinical tests are performed to determine which disease or condition better explains patient's symptoms and signs, such as the test to measure the prostate-specific antigen (PSA) level [63], or the genetic test to identify cystic fibrosis [41], or cellular analysis to detect cell-based diseases such sickle anemia [3], or tests based on medical imaging to ascertain or rule out the presence of breast cancer [59].

Entropy and mutual information have been successfully used many times in medical diagnostics. For example, Richman et al. [64] introduced sample entropy (SampEn) as a method to estimate the entropy of a system represented by a time series, and the technique has been used with success in further researches [1, 21, 54]. In [29] Faes and Porta illustrated a framework to quantify the dynamics of information in coupled physiological systems based on the notion of conditional entropy (CondEn); this method has been used in the neural and cardiovascular time series framework [60, 61]. In [80], Xiong et al. presented a systematic study on the performance, bias, and limitations of three entropy-based measures, to be applied in the context of dynamical systems described by real-world time series, including non-stationarities and long-range correlations. More recently Wiener-Granger causality (WGC) [36, 78], where a variable $X$ Granger causes a variable $Y$ if the information in the past of $X$ improves the prediction of $Y$, was used to analyze time series. Here, $I T$ methods play an important role in the definition of many of the time domain model-free measures of causality [28, 62, 69].

Another relevant application area for IT techniques is medical imaging registration, classification, segmentation and features extraction. Maes et al. [50] reviewed the breakthrough impact of the mutual information maximization criterion in the analysis of multispectral and multitemporal images, where proper image alignment is required to compare corresponding regions in each image volume. Uthoff and Sieren [74] used feature selection methods to quantitatively gauge intensity, texture, and shape of breast lesions; the method is based on three information measures, derived from mutual information, and combined to assess the added benefit of including a feature into the classifying set. A comprehensive review of various image segmentation techniques, also including entropy-derived measures, is given in [18].

Focusing on accuracy measured for diagnostic tests and the agreement between raters, we have to note that the disease is a hidden and objective status of the patient and the physician makes assumptions on it by interpreting the result of the test. Thus, the test is a means to extract information from the patient to diagnose the disease. Therefore, the most accurate diagnostic test will be the one that can extract as much information as possible: the more knowledge flows from the disease to the reader, the more accurate the diagnostic test. The information is implied at the beginning of the diagnostic process and plays a fundamental role.

In this context, physicians have to cope with two primary goals: the first one is to appraise the Diagnostic Test Accuracy (DTA) of a considered test. Such an evaluation also enables clinicians to identify the most effective diagnostic test among a set of possible choices, for instance, comparing digital versus film mammography in diagnosing breast cancer [59]. The second one is to establish an Agreement Measure (AM) to compare evaluations of the same diagnostic outcome produced by different raters or validate new rating systems or devices. For the sake of example, the agreement between ultrasound and automated breast volume scanner can be used to assess breast cancer findings [33].

DTA for dichotomous diagnostic tests has historically been based on the evaluation of sensitivity $(S E)$, specificity $(S P)$, as well as their derived measures, such as likelihood ratios [31, 77]. The main drawback of this approach is that it does not offer a single statistical measure that can summarize the global quality of a dichotomous diagnostic test [58]. The multi-valued case has been handled by selecting a different threshold for $S P$ and then evaluating the area under the curve (AUC) of $S E$ in a Receiver Operating Characteristic $(R O C)$ analysis. In this case too, it is not clear how to compare different tests when they have the same AUC, but a different shape of the ROC curve or what is the best threshold to come back to the dichotomous case, when, for example, a multi-valued ranking scale is used, such as the Breast Imaging-Reporting and Data System (BIRADS) or the Prostate Imaging-Reporting and Data System (PI-RADS).

As for the agreement measures, many different techniques have been introduced so far, but Cohen's $\kappa$ (kappa) [19] is undoubtedly the most popular agreement method between two raters and proved its effectiveness in the last sixty years. Nonetheless, this method suffers from some severe issues: namely, its value is strongly dependent on the prevalence of the disease [68].

Apart from the effectiveness of the methods introduced in the literature to tackle DTA and AM, all the techniques used in practice miss the information's strategic and operative involvement. Since the final purpose of carrying out a diagnostic test is to gain information about the patient's condition, this seems to be a significant shortcoming.

This manuscript is aimed at reviewing the last halfcentury of information theory in assessing the accuracy of diagnostic tests and the agreement between raters, focusing 
on diagnostic test performance measurements and paired agreement evaluation, presenting all the advancements in the field up to the most recent ones. We, first of all, introduce some of the central notions in Shannon's information theory together with the medical diagnostic setting in Section 2. Then, we consider the information measures introduced to gauge the accuracy of diagnostic tests (Section 3) and those meant to evaluate the interrater agreement (Section 4). Section 5 discusses a recent unified approach to both quantify the accuracy of diagnostic tests and, alternatively, assess the agreement between two raters in both dichotomic and multi-valued cases. The effectiveness of this approach is tested in Section 6 by means of two clinical case studies: the first one deals with three raters of a diagnostic test to detect extra-prostatic cancers; the second is related to rapid tests for COVID-19 detection. Finally, Section 7 presents some final remarks and indicates future developments for the topic.

\section{Information theory and medical diagnostics}

From the theoretical point of view, the correct approach to handle information measures is referring to Shannon's Information Theory (IT) [67], which constitutes the mathematical apparatus underlying all current telecommunication systems, based on a rigorous and quantifiable notion of information, over which we obtain some informationderived measures, such as entropy, mutual information (MI) and informational divergence (ID). It is worth noting that Shannon entropy, based on the logarithmic function, has been proved by Khinchin [42] to be the sole measure of information that satisfies some reasonable postulates necessary to define an information measure [2] in a coherent setting.

Before discussing the results presented in medical diagnostics literature during the last fifty years, let us introduce the main actors of Shannon IT, starting with the informational divergence [45], described for the first time a few years after Shannon's work [67]. It has the merit of being the mathematical root over which we can deduce mutual information and entropy in a natural way.

\subsection{Informational divergence, mutual information and entropy}

Let $P=\left\{p_{1}, p_{2}, \ldots, p_{K}\right\}$ and $Q=\left\{q_{1}, q_{2}, \ldots, q_{K}\right\}$ be probability distributions; then

$$
\mathcal{D}(P / / Q) \stackrel{\text { def }}{=} \sum_{i=1}^{K} p_{i} \log \frac{p_{i}}{q_{i}} .
$$

is the informational divergence (ID), or Kullback-Leibler divergence, between the two PDs. ID is always greater than or equal to 0 and the strict equality holds if and only if $P \equiv$ $Q$ [20]. Since $\mathcal{D}(P / / Q)=0$ iff $P \equiv Q$, the divergence can be interpreted as an asymmetric pseudo-distance among probability distributions; it is not a distance because it lacks symmetry and the triangular inequality does not hold in general [20].

Let us now consider the probability distributions $P_{X}$ and $P_{Y}$, associated with the random variables $X$ and $Y$, and the corresponding joint probability distribution $P_{X Y}$. Then the ID

$\mathcal{D}\left(P_{X Y} / / P_{X} P_{Y}\right)=\sum_{x, y} p(x, y) \log \frac{p(x, y)}{p(x) p(y)} \stackrel{\text { def }}{=} I(X, Y)$

can be interpreted as the (oriented) distance from the condition of independence, since $P_{X Y} \equiv P_{X} P_{Y}$ implies ID equal to 0 . The quantity $I(X, Y)$ is the mutual information between the random variables $X$ and $Y$. It is symmetric $(I(X, Y)=I(Y, X))$, and always non-negative, as it is a special kind of informational divergence. So we can interpret $M I$ as a measure of stochastic dependence between two random variables. From the informational point of view, if $I(X, Y)=0$ then $X$ and $Y$ do not exchange information; on the contrary, if $M I$ is greater than 0 , it measures the quantity of information exchanged between the two random variables.

If $Y=X$ we obtain

$$
\begin{aligned}
I(X, X) & =\sum_{i, j} p\left(x_{i}, x_{j}\right) \log \frac{p\left(x_{i}, x_{j}\right)}{p\left(x_{i}\right) p\left(x_{j}\right)} \stackrel{\text { def }}{=} H(X) \\
& =-\sum_{i=1}^{K} p\left(x_{i}\right) \log p\left(x_{i}\right) \geq 0
\end{aligned}
$$

and the quantity defined on the right is the famous Shannon entropy $H(X)$, which expresses the expected value of the random variable $\mathcal{I}(X)=-\log \operatorname{Pr}\{X\}$, which is the self-information [20]. Entropy is the average quantity of information associated with a random variable, and it is simple to verify [20] that

$$
\begin{array}{ll}
0 \leq H(X) \leq \log K \quad\left(=0 \quad \text { iff } P_{X} \text { is degenerative }\right)(4) \\
\left(=\log K \quad \text { iff } P_{X} \text { is uniform }\right)
\end{array}
$$

$$
\begin{aligned}
& I(X, Y)=H(X)-H(X / Y)=H(Y)-H(Y / X) \geq 0 \\
& I(X, Y) \quad \leq \min \{H(X), H(Y)\}
\end{aligned}
$$




\subsection{The medical diagnostics setting}

We can now translate the definitions just seen in terms useful for applications in the field of medical diagnostics. We have a state of disease, or pathologic state for a patient, which is described by the random variable $D$; it takes its value in the set $\mathfrak{D}=\left\{d_{1}, d_{2}, \ldots, d_{K}\right\}$. Similarly, we have a random variable $R$ which represents the report; it is the outcome of a diagnostic test, which is interpreted by a clinician we call rater. $R$ takes its value in the set $\mathfrak{R}=\left\{r_{1}, r_{2}, \ldots, r_{M}\right\}$. We usually assume there are only two mutually exclusive states $D$ of disease for a patient $(K=2)$, either the disease is present $(D=1)$ or absent $(D=0)[58$, 83]; $p_{D}(1)=p(D=1)$ and $p_{D}(0)=p(D=0)$ are the corresponding probabilities. As for the report $R$, we can have several cases. The first one is the dichotomous case, in which there are only two kinds of responses: $R=1$ indicates the presence of the disease, and we call it positive; $R=0$ indicates the absence of the disease, and we call it negative; $p_{R}(1)=p(R=1)$ and $p_{R}(0)=p(R=0)$ are the corresponding probabilities. Another important case is the multi-valued one, as in the breast imaging-reporting and data system (BI-RADS) report, where we can set a 5-point malignancy scale: $1=$ negative; $2=$ benign; $3=$ probably benign; $4=$ suspicious; $5=$ highly suspicious. The last case is that in which the quantity describing the output of the test is continuous, which is the continuous case.

If we restrict our attention to the dichotomous case, the four possible combinations of the considered diagnostic test outcome together with the standard of reference result can be represented by a $2 \times 2$ table known as confusion matrix, which contains the number of true positives (TP), true negatives (TN), false positives $(F P)$ and false negatives $(F N)$ reports. By using these quantities, we can define the following measures:

$$
\begin{array}{cc}
S E \stackrel{\text { def }}{=} p(R=1 / D=1)=\frac{T P}{T P+F N} & F N R \stackrel{\text { def }}{=} p(R=0 / D=1)=\frac{F N}{T P+F N} \\
\text { Sensitivity } & \text { False negative rate } \\
F P R \stackrel{\text { def }}{=} p(R=1 / D=0)=\frac{F P}{F P+T N} & S P \stackrel{\text { def }}{=} p(R=0 / D=0)=\frac{T N}{F P+T N} \\
\text { False positive rate } & \text { Specificity }
\end{array}
$$

where $p(r / d)=p(R=r / D=d)$ is the conditional probability that the report $R$ is $r$, given that the disease variable $D$ equals $d$. Regardless of the patient condition, the diagnosis provided by the diagnostic test is either $R=1$ or $R=0$. Thus, $p(R=1 / D=d)+p(R=0 / D=d)=1$ for all the conditions $d \in\{0,1\}$. The value $p(D=1)$ is the pre-test probability of the disease, while $p(D=1 / R=r)$ is the post-test probability of the disease when the outcome of the diagnostic test is $r \in\{0,1\}$.

\section{Diagnostic information measures and test accuracy}

During the last fifty years, the literature about Shannon information theory in clinical diagnostics has been mainly devoted to gauging the quantity of diagnostic information extracted from clinical tests in specific medical fields.

The first contribution that applied IT techniques to dealing with medical diagnostics seems to be a paper by Good and Card dated 1971 [35]. That work was aimed at maximizing the expected utility of a diagnostic process, where the utility considers both the patient's condition and the various costs associated with the diagnostic process. Since utilities are generally difficult to estimate, the authors suggested some substitutes for them, called quasi-utilities, and they also identified as possible candidates the informational divergence (there called dinegentropy), the mutual information (there called mean information transfer), and the expected weight of evidence. This paper has the merit of introducing, for the first time, "the concept of the patient and doctor as forming a communication or information channel." The authors used the ID $\mathcal{D}(\boldsymbol{q} / / \boldsymbol{\pi})$ to evaluate the stochastic distance between $\boldsymbol{q}=\left\{q_{1}, q_{2}, \ldots, q_{m}\right\}$, which is an estimation, during a diagnostic process, of the probabilities $m$ mutually exclusive diseases $d_{1}, d_{2}, \ldots, d_{m}$, and the initial estimates $\pi$ of the same probabilities, or the vector of initial probabilities. The physician has to estimate the vector $\boldsymbol{q}$ "by means of tests, calculations and judgements." The authors themselves also suggested choosing the test that maximizes $\mathcal{D}(\boldsymbol{q} / / \boldsymbol{\pi})$. Later, they unraveled the connection with the Shannon communication channel and showed that maximizing $\mathcal{D}(\boldsymbol{q} / / \boldsymbol{\pi})$ "comes to the same thing as the maximization of the mean information transfer" [35, page 181]. In the authors' language, this is equivalent to the mutual information $I(\boldsymbol{D}, \boldsymbol{F} / K)$, where $K$ is a conditional variable corresponding to the knowledge of the physician, $\boldsymbol{D}$ is the probability distribution of the diseases $p\left(d_{i}\right)=$ $\pi_{i}$, and $\boldsymbol{F}$ is a vector associated with the chosen test. So, $I(\boldsymbol{D}, \boldsymbol{F} / K)$ has to be maximized by the physician "by choice of the test." Moreover, since $M I$ is the information transmission rate and the expected weight of evidence is the logarithm of a likelihood ratio, the measures are related to the quantity of information extracted by the diagnostic test in both cases.

After this first contribution, there have been several other attempts to introduce Shannon-like methods in medical diagnostics. Another important attempt was the one suggested by Metz, Goodenough, and Rossmann [52], who in 1973 used the mutual information in conjunction with $R O C$ curves. The authors proposed to gauge the imaging system performance by using $R O C$ curve data and, successively, to evaluate radiographic images. This 
approach relates each point of the $R O C$ curve as $1-S P$ varies with the mutual information $I(D, R)$ between the random variables $D$, which represents the two states of disease of the patient, and $R$, which corresponds to the diagnostic report of the reader. This method can be used in two ways: the first one quantifies the maximum amounts of information available on two different $R O C$ curves to compare the quality of the two systems used to generate the curves themselves. The second way measures the quantity of information obtained by a rater operating at any two points of the same $R O C$ curve or a single point on two ROC curves; in this case, the authors measure the information extracted in a diagnostic process by using mutual information. This approach is similar to that of Good and Card [35] even though [52] did not cite it.

In 1978 Okada [56] used MI and a custom-tailored weighted entropy for a slightly different goal: reducing the amount of clinical data by eliminating relatively insignificant items.

The paper [52] by Metz, Goodenough, and Rossman has been a source of inspiration for many subsequent works in several areas of clinical diagnostics. For example, Diamond et al. used the mutual information $I(D, R)$ to gauge the diagnostic effectiveness of different test combinations in the clinical diagnosis of coronary artery disease [24]. The method was compared with an alternative approach which evalutates the average value of the difference between the probability of the disease before and after testing, i.e., $\Delta p=$ $|p(d / r)-p(d)|$. An essential contribution of this paper is that of recognizing the dependency of mutual information from the prevalence of disease; the issue was solved in the Appendix by integrating $M I$ for all the prevalences to obtain an average value to be used for coronary angiography. The subsequent work of Diamond et al. [25] used mutual information to evaluate the information content of the electrocardiographic ST-segment response to exercise relative to the diagnosis of angiographic coronary artery disease.

In paper [65] by Rifkin, the author analyzed the increase of information available for a diagnostic test when one increases the number of outcomes associated with the test results.

Somoza and Mossman [55, 70-73] instead investigated how to choose the best cutoff in diagnostic tests with a continuous response characterized by a ROC curve. Their approach, which extended [52], selected the best cutoff by maximizing the mutual information of the diagnostic test on the $R O C$ curve. The authors used this technique to evaluate the measure of Rapid Eye Movement (R.E.M.) latency as a diagnostic test for depression [70].

In 1990, Asch et al. [6] criticized the use of mutual information, as introduced in [52]; they stated that $M I$ is not able to correctly detect the "prognostic information" that results from the application of a clinical test. They provided an example in which they try to evaluate the information conveyed by a positive test result as a consequence of a change from $p(D=1)=0.1$ of the pre-test probability of disease to $p(D=1 / R=1)=0.9$ of the posttest probability of disease, knowing that we have obtained a positive test result. They evaluated this information by computing the difference $H(D)-H(D / R=1)$, which corresponds to an unweighted sub-component of ordinary mutual information. Since the a priori and a posteriori probabilities are complementary, they obtained a difference equal to 0 . They imputed this to a flaw of information theory since "patients are not indifferent to a chance of disease of $q$ and a chance of $1-q$." They proposed, as an alternative, the use of the difference $p(D=1 / R=1)-p(D=1)$, already discussed in [24], but not mentioned in its bibliography. With abuse of language, they called this approach "Linear information theory" although it deviates from classical Shannon information theory, it wasn't supported by any formal framework, and the word "information," which in their paper denoted a difference between probabilities, was not properly used. The contribution was harshly criticized by Diamond which stated that the example they provided does "not represent a failure of the theory; it represents a failure to appreciate what the theory is about" [23]. This commentary had a reply [7] with a dispute about the concept of "average change of probability" $\Delta p$.

The issue of evaluating the information conveyed by a diagnostic test once a test result is obtained was repurposed by Benish several years later [9]. He suggested replacing the old formula $H(D)-H(D / R=1)$, criticized in [6], with the informational divergence $\mathcal{D}((D / R=1) / / D)$, which solves the pitfall discussed in that paper; it corresponds to measuring the stochastic distance between the post-test and the pre-test probability of disease, knowing that we have obtained a positive test result. The author stressed that it "is not a measure of the absolute amount of information that a test provides." The ID function was proposed in the same year by Lee [46] to select diagnostic tests to rule in or rule out a disease; in this context, the authors suggested the evaluation of $\mathcal{D}((R / D=1) / /(R / D=0)))$ or $\mathcal{D}((R / D=0) / /(R / D=1)))$.

The "absolute amount of information that a test provides," cited in the paper [9] by Benish, has been discussed in two subsequent contributions by the same author $[10,11]$, where it is shown that $I(D, R)$ "quantifies the expected value of the amount of information the test provides." Even though this concept is not new at all e.g., we cited $[24,35,52]$ as prior examples- one has to admit that [11] is the first paper that extensively and systematically described, discussed, and put into a correct environment the problem of measuring the information carried out by a diagnostic test. In this contribution, we can 
also find a quotation of the channel capacity, defined as the maximization of $M I$ across all possible distributions of the pre-test probability of disease; here $M I$ is also used as an index of the performance of a diagnostic test, as the title itself suggests. The use of $I(D, R)$ as the correct tool to manage medical diagnostics information has been supported even from an axiomatic perspective in another paper by Benish [12].

Several other contributions followed these seminal papers, focusing on the practical application of IT methods to actual clinical cases, especially mutual information. For example, in [57] the topic studied is the problem of quantifying the performances of two tests for major depressive disorder, which are the dexamethasone suppression test (DST) and the thyroid-stimulating hormone test (TSH). In contrast, in [4] the authors need to evaluate the effectiveness of GDx nerve fiber analyzer parameters in the diagnosis of glaucoma. In another work [79] the MI is used to extract the most informative mammographic features for breast cancer diagnosis, while the authors of [5] use MI to detect occlusal caries lesions.

Recently, some authors have further developed the old idea - advocated by Good and Card [35] and successively taken up by several other authors - of simply measuring the bare amount of information flowing from the disease to the physician through the use of a diagnostic test. This approach is interesting from the theoretical perspective, but it does not offer the physician an operative tool to compare two diagnostic tests whose accuracy is usually specified through sensitivity and specificity. Moreover, the physician would need a method to measure the global test accuracy using a single number. The first one to pursue this goal was Benish [13], who applied the information theory concept of channel capacity to diagnostic test performance, deriving an expression for channel capacity in terms of test sensitivity and specificity, and finding the prevalence of disease that allows this maximization. It is worth noting that Benish has been the most contributor, during the last 20 years, in the field of application of Shannon information theory to medical diagnostics [9-14].

Subsequently, Girometti and Fabris [32] independently developed an IT framework for diagnostic test accuracy by defining a diagnostic channel that connects the patient disease $\mathfrak{D}$ with the outcome $X$ of the diagnostic test interpreted by the rater $\mathfrak{X}$ (see Fig. 1). While this idea is not new, it properly contextualizes $M I$ usage and formalizes the notion of the diagnostic channel.

The same paper also introduced a normalized measure of the test performance in the interval $[0,1]$ - based on $M I$ as a function of sensitivity and specificity — called the information ratio (IR) of the diagnostic test, which expresses a global measure of the test accuracy and is independent form the prevalence of the disease. Since

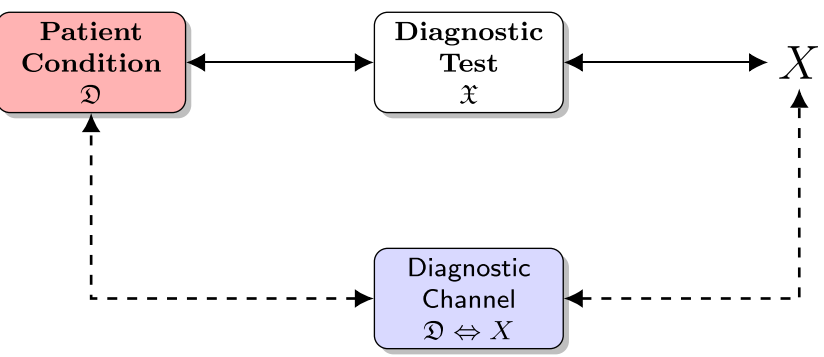

Fig. 1 The diagnostic channel connects the patient disease $\mathfrak{D}$ with the outcome (random variable) $X$ of the diagnostic test interpreted by the rater $\mathfrak{X}$; it is formed by the chain patient condition $\mathfrak{D} \Leftrightarrow$ diagnostic test performed by $\mathfrak{X} \Leftrightarrow X$, and it is briefly indicated as $\mathfrak{D} \Leftrightarrow X$

prevalence is an important variable that can dramatically change the quantity of information measured by the test, and then the quality of the same test, it has been proposed to integrate $M I$ over all the prevalences (e.g., see [24, Appendix]) and normalizing the Area Under the Curve (AUC) with respect to the maximum area available for the standard of reference. A similar method is also discussed for the case of multi-valued diagnostic tests with a variable threshold such as BI-RADS. Section 5 will present this approach.

\section{Informational inter-rater agreement}

The literature about IT methods to evaluate paired agreement is much more limited. To our best knowledge, Klemens was the first one to measure agreement by applying a Shannon-like method. He used a normalized weighted $M I$ as an index of inter-rater agreement [43] and, for each couple of readings $i, j$, the weights $w_{i j}$ were such that $w_{i j}=1$ if $i=j$, and $w_{i j}=0$ if $i \neq j$. This approach is equivalent to taking into account only the cases in which the raters completely agree and decoupling the agreement component of $M I$ from the disagreement part. He then normalized this skewed $M I$ with respect to the sum of the entropies $H(X)$ and $H(Y)$. The paper by Kang et al. [39] uses instead $M I$ to quantify the information shared between outcomes of multiple healthcare surveys. However, this approach dissected $M I$ among the agreement and the disagreement components, too, and it distorted the spirit and the axiomatics of the Shannon's MI function, which averages all the components.

Only recently, Casagrande et al. [15] proposed the use of the classical Shannon orthodox approach also in the agreement context (see also [16]). This is done by introducing an agreement channel, which connects $X$ and $Y$ as the terminals of the chain $X \Leftrightarrow$ diagnostic test performed by $\mathfrak{X} \Leftrightarrow$ patient condition $\mathfrak{D} \Leftrightarrow$ diagnostic test performed by $\mathfrak{Y} \Leftrightarrow Y$, which corresponds to the concatenation of the 
two diagnostic channels $X \Leftrightarrow \mathfrak{D}$ and $\mathfrak{D} \Leftrightarrow Y$ (see Fig. 2); we briefly indicate the agreement channel as $X \Leftrightarrow Y$, and it constitutes the framework to evaluate AM using the socalled informational agreement (IA), which is a normalized measure in the interval $[0,1]$, that can directly be compared with Cohen's $\kappa$.

\section{An IT-based unifying approach}

In this section, we recall the main elements associated with the model we need to measure the performance of a diagnostic test and the agreement between two raters. The starting point is the definition of a diagnostic channel and an agreement channel.

\subsection{Measuring the quality of a diagnostic test}

Based on the literature of the last fifty years, we can now state that mutual information has definitely been accepted as the correct method to measure the quantity of information extracted by a diagnostic test $[11-13,24,32,35,52,73]$. Concerning the medical diagnostics setting Section 2.2, the information exchanged between $D$ and $R$ is measured by

$I(D, R)=\sum_{\substack{d \in \mathfrak{D} \\ r \in \mathfrak{R}}} p(d, r) \log _{2} \frac{p(d, r)}{p(d) p(r)}$

with the logarithm taken to the base 2. Using the Bayes rule $p(d, r)=p(d) p(r / d)$, which is $p(r)=\sum_{d \in \mathfrak{D}} p(d, r)=$ $\sum_{d \in \mathfrak{D}} p(d) p(r / d)$, we have

$$
\begin{aligned}
I(D, R) & =\sum_{\substack{d \in \mathfrak{D} \\
r \in \mathfrak{R}}} p(d) p(r / d) \log _{2} \frac{p(r / d)}{p(r)} \\
& =\sum_{\substack{d \in \mathfrak{D} \\
r \in \mathfrak{R}}} p(d) p(r / d) \log _{2} \frac{p(r / d)}{\sum_{d^{\prime} \in \mathfrak{D}} p\left(d^{\prime}\right) p\left(r / d^{\prime}\right)}
\end{aligned}
$$

In the dichotomous case, the prevalence of the disease $P_{D} \stackrel{\text { def }}{=} p(D=1)$ equals $1-p(D=0)$, because $p(d)$ is a probability. Hence, $p(r)=\sum_{d^{\prime} \in \mathfrak{D}} p\left(d^{\prime}\right) p\left(r / d^{\prime}\right)$ can be rephrased as $p(r / D=0)+p(D=1)(p(r / D=1)-$ $p(r / D=0))$. Moreover, $p(r=1 / d)=1-p(r=0 / d)$ for any $d$, thus, due to $S E$ and $S P$ definitions in terms of $p(r / d)$ (see Eq. 6), the mutual information between $D$ and $R$ equals

$$
\begin{aligned}
I(D, R)= & P_{D}\left(\log _{2}(1-S E)+S E\left(\log _{2} S E-\log _{2}(1-S E)\right)\right) \\
& +\left(1-P_{D}\right)\left(\log _{2}(1-S P)+S P\left(\log _{2} S P-\log _{2}(1-S P)\right)\right) \\
& -\left((1-S P)+P_{D}(S E+S P-1)\right) \log _{2}[(1-S P) \\
& \left.+P_{D}(S E+S P-1)\right]-\left(S P+P_{D}(1-(S E+S P))\right) \log _{2}[S P \\
& \left.+P_{D}(1-(S E+S P))\right]
\end{aligned}
$$

Equation 9 proves that the mutual information between the rater and the disease exclusively depends on $S E, S P$, and $P_{D}$. On the one hand, this measure is subject to the prevalence, which is not always known and may be biased; on the other hand, once $S E$ and $S P$ are measured, we can evaluate the mutual information itself for any possible prevalence by using Eq. 9. In order to stress this last aspect, we may refer to the mutual information between $D$ and $R$ - i.e., $I(D, R)$ - also as $M I_{S E, S P}\left(P_{D}\right)$ or, whenever both $S E$ and $S P$ can be deduced from the context, as $M I\left(P_{D}\right)$.

In order to define a prevalence-independent metric for rater performances, we can account for all the possible mutual information values for any prevalence, which is done by integrating $M I$ over all the prevalences of disease in the interval $[0,1][24,32]$

$\overline{M I} \stackrel{\text { def }}{=} \int_{0}^{1} M I\left(P_{D}\right) \mathrm{d} P_{D}$

This corresponds to evaluating the AUC of the MI curve over all the prevalences. The $M I$ curve associated with the case $S E=1$ and $S P=1$ is the one having the greatest admissible AUC. This $M I$ curve is the standard of reference (SR) and its AUC equals $\overline{M I}_{1,1}=1 / \ln 4$. Figure 3 depicts the standard of reference $M I_{1,1}$ together with the curves $M I_{0.5,1}(S E=0.5$ and $S P=1)$ and $M I_{1,0.5}(S E=1$ and $S P=0.5)$ as the prevalence of the disease varies in the closed interval $[0,1]$. Note that when the curves intersect, as

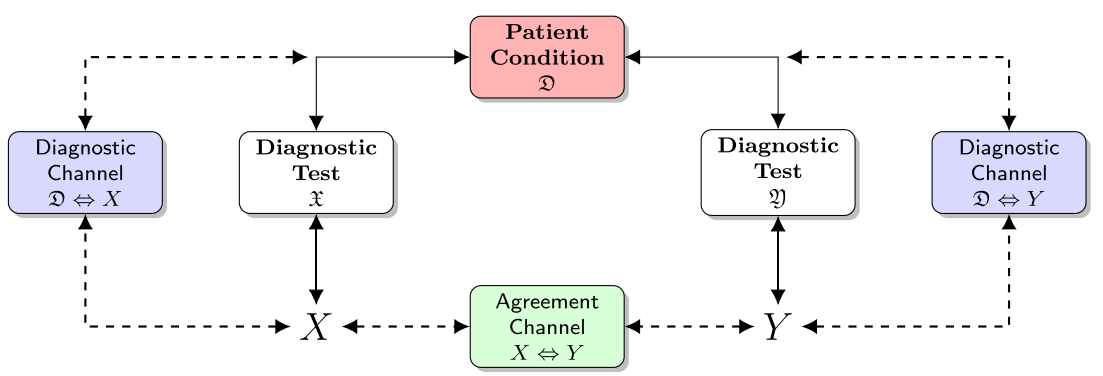

Fig. 2 The diagnostic channel connects the patient disease $\mathfrak{D}$ with the outcome (random variable) $X(Y)$ of the diagnostic test interpreted by the rater $\mathfrak{X}(\mathfrak{Y})$; it is formed by the chain patient condition $\mathfrak{D} \Leftrightarrow$ diagnostic test performed by $\mathfrak{X} \Leftrightarrow X$, and it is briefly indicated as $\mathfrak{D} \Leftrightarrow X$.
The agreement channel connects the random variables $X$ and $Y$, that express the raters outcomes. They are the terminals of the chain $X \Leftrightarrow$ diagnostic test performed by $\mathfrak{X} \Leftrightarrow$ patient condition $\mathfrak{D} \Leftrightarrow$ diagnostic test performed by $\mathfrak{Y} \Leftrightarrow Y$. It is briefly indicated as $X \Leftrightarrow Y$ 


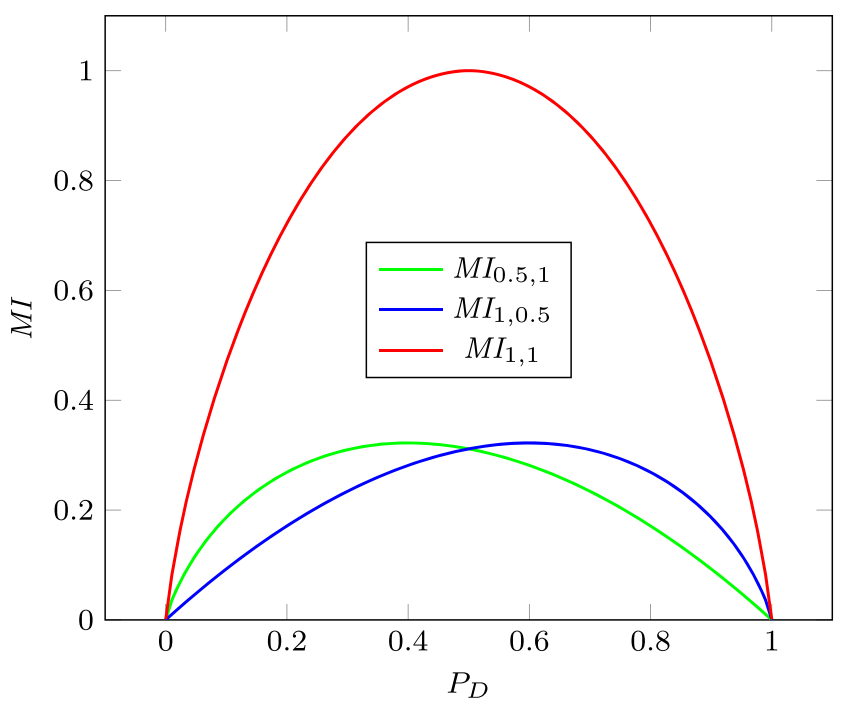

Fig. $3 M I_{0.5,1}(S E=0.5$ and $S P=1), M I_{1,0.5}(S E=1$ and $S P=0.5)$, and $M I_{1,1}(S E=1$ and $S P=1)$ as the prevalence of the disease varies in $[0,1]$

in the example of Fig. 3, one can specify the interval with the best behavior for each curve. In this case, we have the green curve better for $P_{D}<0.5$ and the blue one for $P_{D}>0.5$.

The information ratio $I R$ [32] is $\overline{M I}$ normalized with respect to the maximum value of it, i.e., $\overline{M I}_{1,1}$,

$I R \stackrel{\text { def }}{=} \frac{\overline{M I}}{\overline{M I}_{1,1}}=\ln 4 \int_{0}^{1} M I\left(P_{D}\right) \mathrm{d} P_{D}$.

It is worth to notice that the value of $I R$ still depends on both $S E$ and $S P$.

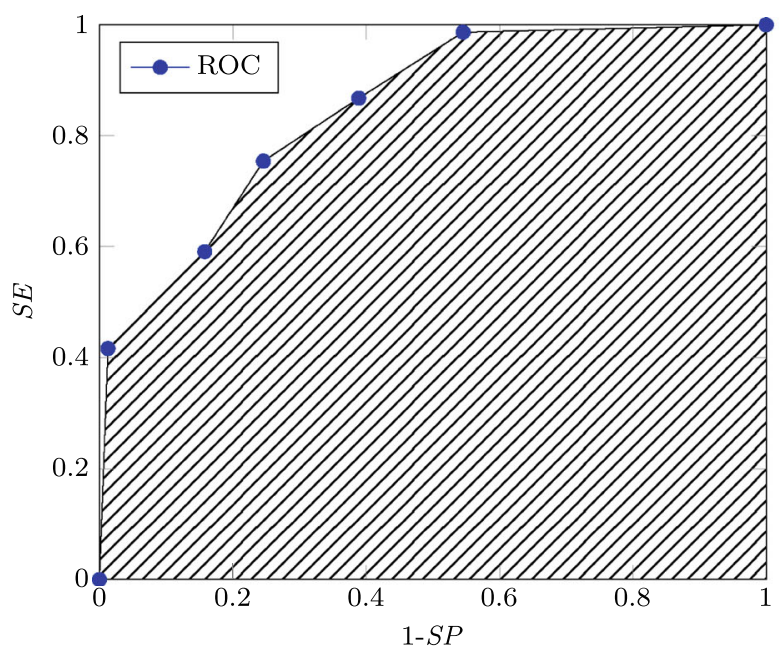

(a) A classical $R O C$ curve for a 7-points malignancy scale. The AUC of $R O C$ curve depicted in this figure is about 0.848 .

Fig. 4 Two purely illustrative ROC and GIR curves for a 7-point malignancy scale
As far as the multi-valued case is concerned, we can refer again to [32], where the global quality of the test is evaluated by changing the threshold of $S P$, so as to obtain an $I R$ value for each value of $1-S P$. In Fig. 4a we can see an example of a classical $R O C$ curve for a 7-point BIRADS test. The corresponding information ratio curve IRC is shown in Fig. 4b; we have an $I R$ value for each threshold $1-S P$ and the AUC of the curve is related with the limit information curve LIC, drawn by fixing $S E=1$ for all values of $1-S P$, which corresponds to the curve associated with the maximum amount of information we can gain for each value of $1-S P$. The AUC of the LIC curve is computed in [32] and is equal to $2-\pi^{2} / 6 \approx 0.35506$. The normalization of the IRC's AUC with respect to the AUC of the LIC curve gives the global information ratio GIR

$G I R \stackrel{\text { def }}{=} \frac{A U C_{I R C}}{2-\pi^{2} / 6}$

which expresses a global, prevalence-independent, normalized evaluation of the quality of a multi-valued diagnostic test. It can be thought of as the information counterpart of a ROC curve.

\subsection{Measuring the agreement between raters}

The problem of evaluating the agreement between two raters is solved by using the approach depicted in [15], which consists in expressing it as the quantity of information flowing through the agreement channel of Fig. 2, which is

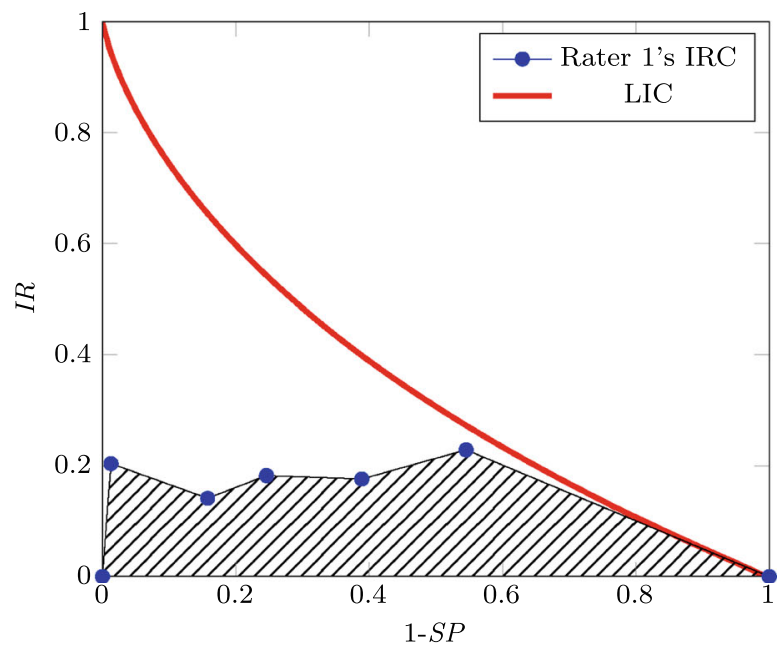

(b) The corresponding IRC curve for the same 7-points malignancy scale. The GIR of the IRC depicted in this figure is about 0.422 . 
the virtual channel connecting the random variables $X$ and $Y$ through the information path $X \Longrightarrow$ rating by $\mathfrak{X} \Longrightarrow$ condition $\mathfrak{D} \Longrightarrow$ rating by $\mathfrak{Y} \Longrightarrow Y$. It is so because $I(X, Y)$ is a measure of the stochastic dependence between $X$ and $Y$. Since $I(X, Y) \leq \min \{H(X), H(Y)\}$ (see Eq. 5), we can normalize $I(X, Y)$ with respect to $\min \{H(X), H(Y)\}$; this leads to the informational agreement $I A$

$I A(X, Y) \stackrel{\text { def }}{=} \frac{I(X, Y)}{\min \{H(X), H(Y)\}}$

whose value ranges in the interval $[0,1]$. As pointed out in [15], contrary to what happens with Cohen's $\kappa, I A$ correctly measures the stochastic distance between $P_{X Y}$ and $P_{X} P_{Y}$, which is the distance of the two raters from the condition of independence. This means that $I A$ gauges the (normalized) amount of information exchanged between the two raters. Furthermore, this measure can be used in both the dichotomic and multi-valued scale ratings.

\section{Two case studies from clinical diagnostics}

To assess the global quality of the approaches depicted in Section 5, which is to measure the quality of the readings of some raters that have to evaluate the outcomes of a diagnostic test and the mutual agreement between a couple of raters, we considered two case studies; the first one deals with raters of a diagnostic test to detect extra-prostatic cancers; the second one is related to COVID-19 rapid detection.

\subsection{Detecting extra-prostatic cancers}

For this analysis, we took the original data set used with the paper [82]. In this study, investigators assessed whether Magnetic Resonance Imaging (MRI) of the prostate added value to clinical models in diagnosing so-called pathological stage $\geq \mathrm{T} 3$ prostate cancer, i.e., cancer with extra-prostatic extension into surrounding soft tissue and invasion of the seminal vesicles at pathological analysis after surgery. Preoperative knowledge of stage $\geq \mathrm{T} 3$ is essential to both plan the type of surgery - for instance, to plan whether to perform nerve-sparing surgery- and predict the risk of recurrent prostate cancer after primary treatment.

In the source study, three different radiologists with 8,6 , and 2 years of experience in prostate MRI (raters $\mathcal{R}_{1}, \mathcal{R}_{2}$ and $\mathcal{R}_{3}$, respectively) prospectively evaluated MRI examinations performed to stage prostate cancer before radical prostatectomy. They attributed an MRI stage on a rank scale (T1c, T2a, T2b, T2c, T3a, and T3b). On this basis, we have performed three kinds of analysis. The first one was devoted to testing the diagnostic accuracy of each radiologist in assessing pathological stage $\geq \mathrm{T} 3$ under the form of the $I R$. In order to achieve this goal, MRI readings were dichotomized by assuming that the MRI stage $\geq \mathrm{T} 3 \mathrm{a}$ was the cutoff for the pathological stage $\geq \mathrm{T} 3$ diagnosis.

In the second analysis, we evaluated readers' accuracy in diagnosing pathological stage $\geq \mathrm{T} 3$ on a multi-valued basis, i.e., by obtaining the $G I R$ and $R O C$ curves built upon all the rank values attributed by radiologists in image analysis.

Lastly, we focused on assessing pairwise inter-rater agreement - i.e., $\mathcal{R}_{1}$ vs $\mathcal{R}_{2}, \mathcal{R}_{1}$ vs $\mathcal{R}_{3}$, and $\mathcal{R}_{2}$ vs $\mathcal{R}_{3}$. This has been done by computing the information agreement for both dichotomic and multi-valued cases and Cohen's $\kappa$ for the dichotomic case alone.

\subsubsection{Results for extra-prostatic cancers}

Table 1 shows the $I R$ of the three raters with respect to the standard of reference for the data set associated with the search of extra-prostatic cancers; it also contains the parameters usually computed to determine the quality of a diagnostic test, which are essentially sensitivity, specificity, false positive and false negative rates. Figure 5 shows instead the variation of $M I$ with respect to the prevalence of the disease.

Figure 6 contains the $I R$ profile while changing the $S P$ threshold, so as to generate the GIR curve and the corresponding normalized value. The third point starting from the left corresponds to the standard cutoff $1-4 \mid 5-6$ - to be intended as surgery is required from rate 5 on - we can appreciate that in all the three cases it has the maximum value of $I R$; this means that the threshold used is the best possible since it carries the maximum amount of diagnostic information.

The results of the standard $R O C$ analysis are reported in Fig. 7. It is not clear how to validate the best threshold for the $R O C$ curve.

As for the agreement comparison, the results are available in Table 2; we have evaluated the IA for the dichotomous and the multi-valued cases and Cohen's $\kappa$ for the dichotomous case alone.

Table 1 The sensitivity, specificity, false positive, false negative rates, and the value of $I R$ for each of the extra-prostatic cancer raters $\mathcal{R}_{1}$, $\mathcal{R}_{2}$, and $\mathcal{R}_{3}$ with respect to the standard of reference

\begin{tabular}{llll}
\hline & $\mathcal{R}_{\mathbf{1}}$ & $\mathcal{R}_{\mathbf{2}}$ & $\mathcal{R}_{\mathbf{3}}$ \\
\hline$S E$ & 0.62 & 0.67 & 0.58 \\
$S P$ & 0.82 & 0.73 & 0.88 \\
$F N R$ & 0.38 & 0.33 & 0.42 \\
$F P R$ & 0.18 & 0.27 & 0.12 \\
$I R$ & 0.142 & 0.112 & 0.167 \\
\hline
\end{tabular}




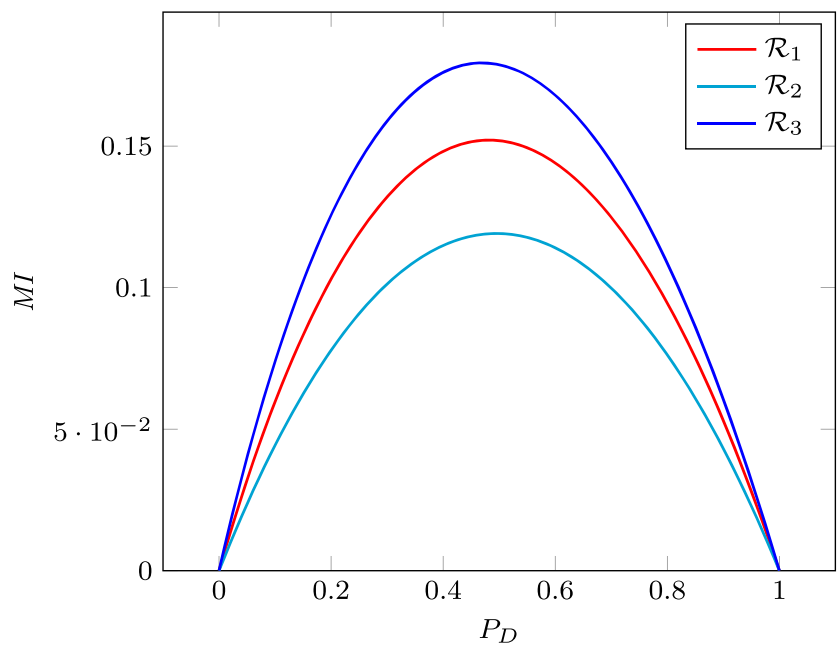

Fig. 5 The mutual information of each of the extra-prostatic cancer raters $\mathcal{R}_{1}, \mathcal{R}_{2}$, and $\mathcal{R}_{3}$ against the standard of reference as the prevalence of the disease varies in the interval $[0,1]$

\subsubsection{Discussion on extra-prostatic cancers}

Table 1 shows that the $I R$ s of $\mathcal{R}_{1}, \mathcal{R}_{2}$, and $\mathcal{R}_{3}$ are 0.142 , 0.112 , and 0.167 , respectively. Since they lay within a narrow range, the three raters are almost equivalent, with $\mathcal{R}_{3}$ performing a little better than the others and $\mathcal{R}_{1}$ is the second best. Figure 5 shows that this ordering holds for all the prevalences, because the $M I$ curve related to $\mathcal{R}_{3}$ is always above those of the other two raters, while that associated to $\mathcal{R}_{2}$ is below the curve of $\mathcal{R}_{1}$ for all the possible prevalences.

The $I R$ s of all the raters are quite small in absolute terms with respect to the theoretical maximum values for $I R$, i.e., 1. While this is partially due to both the low sensitivity (0.62, 0.67, and 0.58, respectively), which is typical for this kind of measure, and the not so high specificity $(0.82$, 0.73 , and 0.88 , respectively) of the raters, this drift is quite frequent in the general case for informational measures that, being based on entropy, are able to discriminate even modest changes in rater performances when both sensitivity and specificity are close to 1 .

Figure 6 shows the GIR diagrams of the three raters together with the corresponding values - i.e., 0.208, 0.225, and 0.190 for $\mathcal{R}_{1}, \mathcal{R}_{2}$, and $\mathcal{R}_{3}$, respectively. Also, in this case, we can consider the quality of the raters almost equivalent, but with a different ordering as $\mathcal{R}_{2}$ performs better than the other two and $\mathcal{R}_{1}$ follows. It is worth noticing that this last ordering seems to be more tuned with the one subtended by the experience of the three raters - i.e., $\mathcal{R}_{1}$ in first place, $\mathcal{R}_{2}$ in second, and $\mathcal{R}_{3}$ in third. This seems to suggest that the GIR analysis, based on a variation of the threshold for specificity, is more coherent than the simple $I R$ analysis based on a fixed threshold.

The AUC under the ROC curves of Fig. 7 offers a slightly different and less convincing vision of the raters' performance, since in this case the most experienced rater $\mathcal{R}_{1}$, having 8 years of experience in prostate $\mathrm{MRI}$, is considered the worst rater with $\mathcal{R}_{2}$ topping the other two.

The agreement analysis, interestingly, offers support to the idea that $\mathcal{R}_{2}$ and $\mathcal{R}_{3}$ are the furthest away, as specified in $I R$ and GIR analysis, since for all three methods used, (dichotomous $I A$, multi-valued $I A$ and Cohen's $\kappa$, see Table 2) it comes out that $\mathcal{R}_{2}$ vs $\mathcal{R}_{3}$ shows the worst value of the agreement. Since the $I A$ for the multivalued scale is by far the most refined method from the theoretical point of view, we can accept the fact that $\mathcal{R}_{1}$ vs $\mathcal{R}_{2}$ have the best agreement, also because the other two methods, dichotomous $I A$ and $\kappa$, would suggest that the best agreement is between $\mathcal{R}_{1}$ and $\mathcal{R}_{3}$, which seems not coherent with the scale of years of experience of the raters.

In conclusion, we could suggest that $\mathcal{R}_{2}$ is the best rater among the three, $\mathcal{R}_{1}$ comes in second place, and $\mathcal{R}_{3}$ is by far the worst of the three. In this sense the GIR and the multivalued $I A$ appear to be the best tools to use when evaluating the quality of a reader or the agreement between readers, at least when we have a multi-valued scale of ratings.

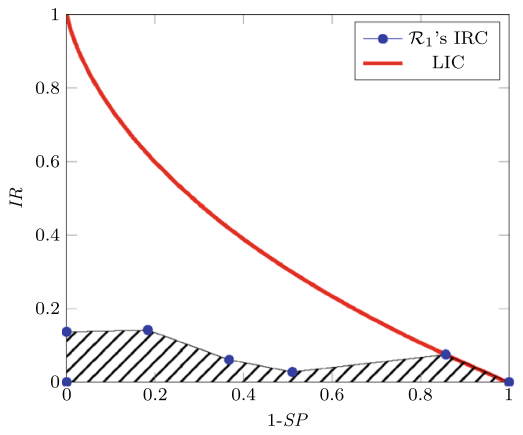

(a) $\mathcal{R}_{1}$ GIR analysis: the $G I R$ is about 0.208

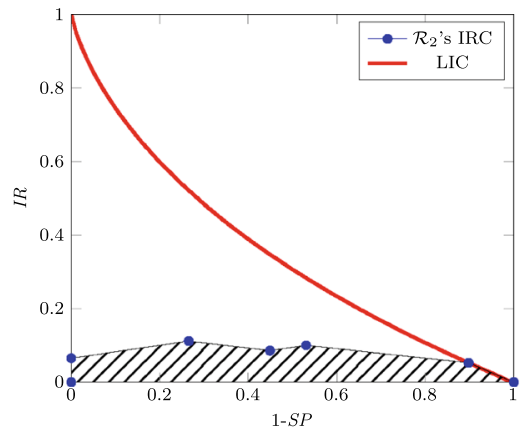

(b) $\mathcal{R}_{2}$ GIR analysis: the GIR is about 0.225 .

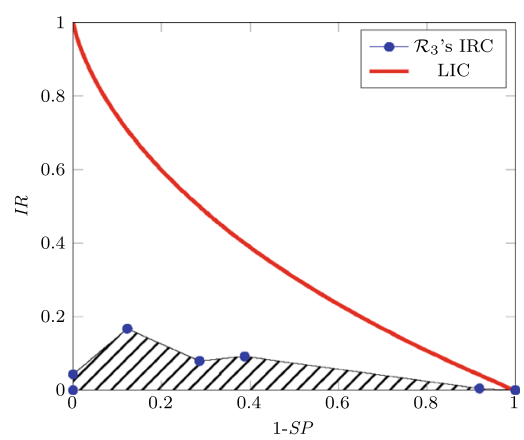

(c) $\mathcal{R}_{3}$ GIR analysis: the GIR is about 0.190 .

Fig. 6 GIR analysis of the three extra-prostatic cancer raters versus the standard of reference 


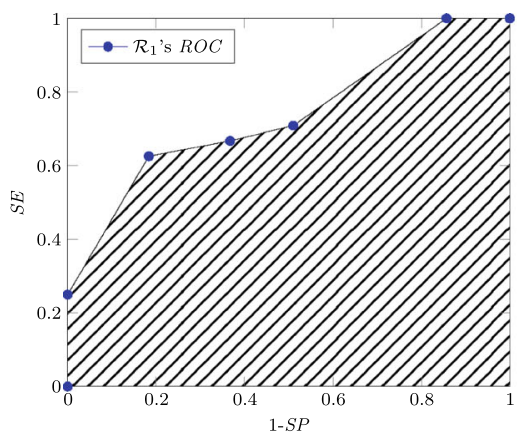

(a) $\mathcal{R}_{1} R O C$ analysis: the AUC is about 0.736 .

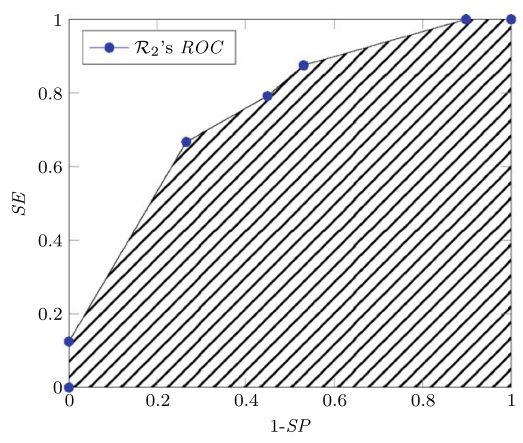

(b) $\mathcal{R}_{2} \quad R O C$ analysis: the AUC is about 0.753 .

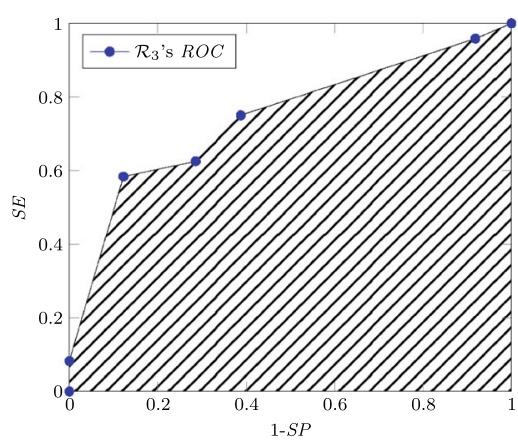

(c) $\mathcal{R}_{3} \quad R O C$ analysis: the AUC is about 0.743 .

Fig. 7 ROC analysis of the three extra-prostatic cancer raters versus the standard of reference

\subsection{Evaluating the effectiveness of serology tests for COVID-19 detection}

A possible application for the analysis described in Section 5.1 is the comparison of the accuracy of COVID19 tests. For the sake of example, we considered the data reported in [53] and we analyzed the comparison between RT-PCR, which is the standard of reference for COVID19 diagnosis, and two automated and one rapid lateral flow immunoassays for the detection of anti-SARS-CoV2 antibodies. These essays highlight SARS-CoV-2 specific antibodies in blood samples and allow rapid identification of the COVID-19 disease in the considered subjects. We limited our analysis to the Euroimmun Anti-SARS-CoV2 ELISA IgG and IgA combined assays (Euroimmun, Luebeck, Germany), the Maglumi ${ }^{\mathrm{TM}} 2019-\mathrm{n}-\mathrm{Cov}$ IgG and IgM combined immunoassays (CLIA), and the 2019-n$\mathrm{CoV}$ IgG/IgM combined rapid test cassette (LaboOn Time) (LabOn Time, Bio Marketing Diagnostics, or Akiva, Israel).

By using the number of true positives, RT-PCR positive, true negative, and negative RT-PCR of the considered combined tests, that are reported in [53, Table 1], we calculated the sensitivities and specificities of the assays. Then, their $I R$ s were assessed and the tests were sorted according to their IRs to identify the most accurate test on average over all possible prevalences of disease. Furthermore, we computed the mutual information of the investigated tests and RT-PCR for the values $1 / 8,2 / 8, \ldots$, and $7 / 8$ of the prevalences of disease by using Eq. 9 . For each of these prevalences, we re-sorted the tests according to their mutual information with respect to the standard of reference and we established the more effective tests among those analyzed for the specific value of $P_{D}$.

\subsubsection{Results of the analysis of COVID-19 tests}

Table 3 shows the values of $I R$ for the tests ELISA, CLIA and LaboOn Time, together with the corresponding sensitivity, specificity, false positive and false negative rates.

Figure 8 shows the corresponding $M I$ curves as a function of the prevalence of disease. Note that the ELISA and CLIA curves intersect for $P_{D} \approx 0.55$.

Table 4 reports the mutual information of the considered COVID-19 antibodies tests versus the standard of reference as the prevalences of the disease varies in $\{1 / 8,2 / 8, \ldots, 7 / 8\}$.

\subsubsection{Discussion on the COVID-19 tests}

As far as the analysis of the COVID-19 antibodies tests is concerned, the $I R \mathrm{~s}$ reported in Section 6.2.1 suggests that, if there are no preferences to the ability to identify positive cases with respect to the capability of discharging the negative ones, whenever the prevalence of the disease

Table 2 Agreement between each pair of extra-prostatic cancer raters $\mathcal{R}_{1}$ vs $\mathcal{R}_{2}, \mathcal{R}_{1}$ vs $\mathcal{R}_{3}$, and $\mathcal{R}_{2}$ vs $\mathcal{R}_{3}$, both in the dichotomous and in the multi-valued case, expressed by the $I A$. The last column contains Cohen's $\kappa$ values

\begin{tabular}{llll}
\hline Raters & IA dichotomous & IA multi-valued & Cohen's $\kappa$ \\
\hline $\mathcal{R}_{1}$ vs $\mathcal{R}_{2}$ & 0.259 & 0.361 & 0.558 \\
$\mathcal{R}_{1}$ vs $\mathcal{R}_{3}$ & 0.490 & 0.337 & 0.741 \\
$\mathcal{R}_{2}$ vs $\mathcal{R}_{3}$ & 0.222 & 0.263 & 0.487 \\
\hline
\end{tabular}


Table 3 Sensitivity, specificity, false positive, false negative rates and the value of IR for the Euroimmun Anti-SARS-CoV-2 ELISA IgG and IgA combined assays, Maglumi ${ }^{\mathrm{TM}}$ 2019-n-Cov IgG and IgM combined immunoassays (CLIA) and LaboOn Time kit

\begin{tabular}{llll}
\hline & ELISA & CLIA & LaboOn Time \\
\hline$S E$ & 0.844 & 0.631 & 0.719 \\
$S P$ & 0.875 & 1.000 & 1.000 \\
$F N R$ & 0.156 & 0.369 & 0.281 \\
$F P R$ & 0.125 & 0.000 & 0.000 \\
$I R$ & 0.392 & 0.417 & 0.504 \\
\hline
\end{tabular}

is unknown, LaboOn Time is preferable to the other two tests for all the values of prevalence, with CLIA in second position among the three.

Moreover, Table 4 indicates that CLIA performs better than ELISA for all the preferences in $\{1 / 8,2 / 8,3 / 8,4 / 8\}$, but the latter is more accurate than the former for the prevalences $5 / 8,6 / 8$, and $7 / 8$. This is also visible in Fig. 8 where the mutual information curves of these two tests and RT-PCR intersect on $P_{D} \approx 0.55$.

\section{Discussion and conclusions}

Information theory has been used in many areas, such as computer science, physics, biology, linguistics, taxonomy, psychology and many others. It has been applied also in medical diagnostics, for example, to study systems represented by a time series, or to describe the dynamics of information in coupled physiological systems, or to extract features in medical imaging registration, classification and segmentation.

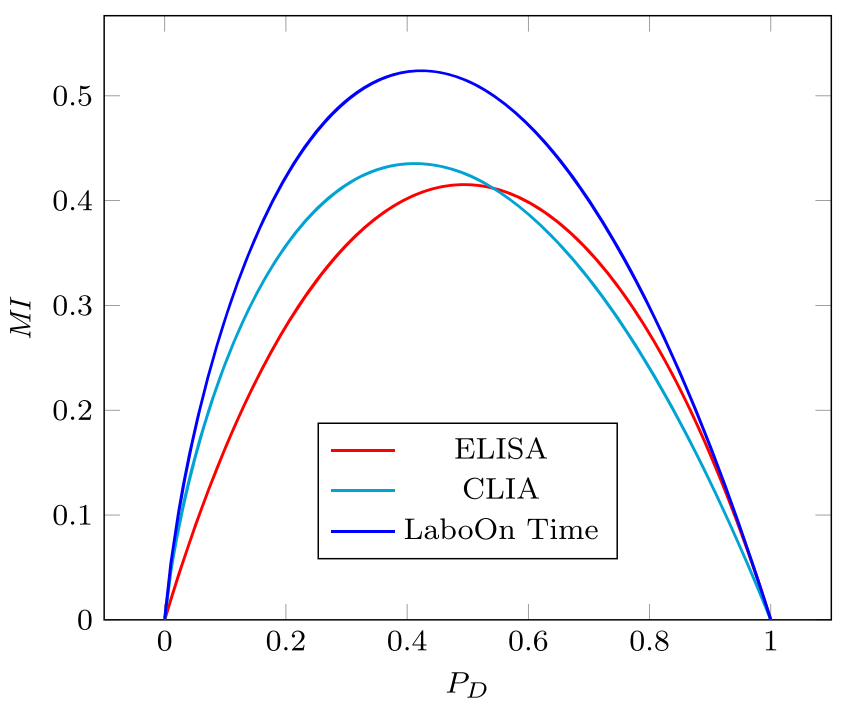

Fig. 8 The $M I$ curves of the considered COVID-19 antibodies tests as a function of the prevalence of disease
As for the problem of assessing the accuracy and agreement of diagnostic tests, many intriguing results have been obtained in the last fifty years. Nevertheless, even though these contributions are based on consolidated mathematical tools [67], they have not been considered for daily clinical practice, which instead keeps employing, in both DTA and AM contexts, more classical approaches. This discrepancy may be due to several reasons.

In some cases, the proposed methods merely used Shannon functions, such as entropy, mutual information, and informational divergence, as flat formulas to derive different custom-modified measures to express DTA or AM. This approach, when not supported by any axiomatic framework, led to both questionable and difficult-to-beinterpreted results.

In other cases, even though the suggested measures remained inside the orthodoxy depicted by Shannon, their advantages with respect to the mainstream statistical approaches, such as the commonly used Cohen's $\kappa$, remained obscure to the vast audience of physicians partially because of the lack of the necessary software tools to broadly test and, possibly, adopt them. Perhaps, the most important motivation for not using Shannon-derived measures for DTA and AM in medical diagnostics is that they have seldom been operatively compared with the tools daily used in clinical diagnostic.

The path depicted in $[15,16,32]$ tries to overcome all these limitations. Clinical tests are modeled as a channel (the diagnostic channel) that routes information about the disease from patients to their diagnosticians and, because of this, Shannon-theory can be applied to evaluate a normalized measure of the information acquired by using the tests themselves in both dichotomic and multi-valued cases [32]. Analogously, the agreement channel between pairs of raters is used to gauge the quantity of information virtually exchanged by raters themselves in their evaluations and, as a consequence, their agreement $[15,16]$. The comparisons of the proposed measures against the standard dogmatic statistical tools, such as Cohen's $\kappa$, Scott's $\pi$, or Bangdiwala's $B$, suggested that the former perform better than the latter in both cited tasks. 
Table 4 The mutual information of three COVID-19 antibodies tests versus the standard of reference, i.e., RT-PCR, as the prevalences of the disease varies in the set $\{1 / 8,2 / 8, \ldots, 7 / 8\}$ (see Section 6.2)

\begin{tabular}{llllllll}
\hline Tests & $\mathbf{P}_{\mathbf{D}}=\mathbf{0 . 1 2 5}$ & $\mathbf{P}_{\mathbf{D}}=\mathbf{0 . 2 5 0}$ & $\mathbf{P}_{\mathbf{D}}=\mathbf{0 . 3 7 5}$ & $\mathbf{P}_{\mathbf{D}}=\mathbf{0 . 5 0 0}$ & $\mathbf{P}_{\mathbf{D}}=\mathbf{0 . 6 2 5}$ & $\mathbf{P}_{\mathbf{D}}=\mathbf{0 . 7 5 0}$ & $\mathbf{P}_{\mathbf{D}}=\mathbf{0 . 8 7 5}$ \\
\hline ELISA & 0.197 & 0.323 & 0.393 & 0.415 & 0.389 & 0.316 & 0.190 \\
CLIA & 0.280 & 0.392 & 0.433 & 0.425 & 0.374 & 0.286 & 0.161 \\
LabOn & 0.329 & 0.465 & 0.519 & 0.514 & 0.457 & 0.353 & 0.202 \\
\hline
\end{tabular}

So, why are these Shannon-oriented measures still far away from being widely adopted? On the one hand, software tools that allow non-experts in information theory to evaluate these metrics are still missing; this aspect discourages physicians from using the discussed approach in their research manuscripts and standard practices and it delays the penetration of the information theory tools in the clinical community. On the other hand, the absence of any absolute qualitative reference scale for the new metrics plays a role in this lack of interest too. In other terms, no scale that establishes whether one can consider an $I R, G I R$, or $I A$ value to be "good" or "bad" has been proposed yet. It is worth noticing that, even in the context of classical statistical tools, these scales are either missing or, in the best case, totally arbitrary and devoid of any objective foundation, such as the widespread-adopted linear scale proposed in [51] to rate Cohen's $\kappa$ - i.e., [0, 0.2)(“none to slight"), [0.2, 0.4) ("fair"), [0.4, 0.6) ("moderate"), $[0.6,0.8)$ ("substantial"), and $[0.8,1.0)$ ("perfect or almost perfect agreement").

We feel confident in foretelling that the mentioned obstacles to the adoption of the IT-based evaluation approach in the clinical domain will be removed in the next years, which will release new advances in medical diagnostics.

Acknowledgements The authors would like to thank the anonymous reviewers whose comments/suggestions helped improve and clarify the manuscript.

Funding This work has been partially supported by the Istituto Nazionale di Alta Matematica (INdAM) and by the project Dipartimento di Eccellenza of the Dept. of Mathematics and Geosciences of the University of Trieste.

Open Access This article is licensed under a Creative Commons Attribution 4.0 International License, which permits use, sharing, adaptation, distribution and reproduction in any medium or format, as long as you give appropriate credit to the original author(s) and the source, provide a link to the Creative Commons licence, and indicate if changes were made. The images or other third party material in this article are included in the article's Creative Commons licence, unless indicated otherwise in a credit line to the material. If material is not included in the article's Creative Commons licence and your intended use is not permitted by statutory regulation or exceeds the permitted use, you will need to obtain permission directly from the copyright holder. To view a copy of this licence, visit http://creativecommons. org/licenses/by/4.0/.

\section{References}

1. Aboy M, Cuesta-Frau D, Austin D, Mico-Tormos P (2007) Characterization of sample entropy in the context of biomedical signal analysis. In: 2007 29th Annual international conference of the IEEE engineering in medicine and biology society. IEEE, pp 5942-5945

2. Daróczy J, Aczél Z (1975) On measures of information and their characterizations. Mathematics in science and engineering, vol 115. Academic Press, New York

3. Arishi WA, Al-Hadrami HA, Zourob M (2021) Techniques for the detection of sickle cell disease: a review. Micromachines 12(5):519

4. Arslan U, Bozkurt B, Karaağaoğlu AE, Irkec MT (2011) Evaluation of GDx parameters by using information theory. Turkish $\mathrm{J}$ Med Sci 41(1):117-124. https://doi.org/10.3906/sag-0909-284

5. Arslan U, Karaağaoğlu AE, Özkan G., Kanli A (2014) Evaluation of diagnostic tests using information theory for multiclass diagnostic problems and its application for the detection of occlusal caries lesions. Balkan Medi J 31:214-218. https://doi.org/10.5152/balkanmedj.2014.13218

6. Asch DA, Patton JP, Hershey JC (1990) Knowing for the sake of knowing: the value of prognostic information. Med Decis Making 10:47-57. https://doi.org/10.1177/0272989X9001000108

7. Asch DA, Patton JP, Hershey JC (1991) Prognostic information versus accuracy: once more with meaning. Med Decis Making 11:45-47. https://doi.org/10.1177/0272989X9101100108

8. Attneave F (1959) Applications of information theory to psychology: a summary of basic concepts, methods, and results. A Holt-Dryden Book. Holt. https://books.google.it/books? $\mathrm{id}=\mathrm{VnB} 9 A \mathrm{AAAMAAJ}$

9. Benish WA (1999) Relative entropy as a measure of diagnostic information. Med Decis Making 19:202-206. https://doi.org/10.1177/0272989X9901900211

10. Benish WA (2002) The use of information graphs to evaluate and compare diagnostic tests. Methods Inf Med 41:114-118. https://doi.org/10.1055/s-0038-1634294

11. Benish WA (2003) Mutual information as an index of diagnostic test performance. Methods Inf Med 42(3):260-264. https://doi.org/10.1055/s-0038-1634358

12. Benish WA (2009) Intuitive and axiomatic arguments for quantifying diagnostic test performance in units of information. Methods Inf Med 48(6):552-557. https://doi.org/10.3414/ME0627

13. Benish WA (2015) The channel capacity of a diagnostic test as a function of test sensitivity and test specificity. Stat Methods Med Res 24(6):1044-1052. https://doi.org/10.1177/0962280212439742

14. Benish WA (2020) A review of the application of information theory to clinical diagnostic testing. Entropy, 22. https://doi.org/10.3390/e22010097

15. Casagrande A, Fabris F, Girometti R (2020) Beyond kappa: an informational index for diagnostic agreement in dichotomous and multivalue ordered-categorical ratings. Med Biol Eng Comput 58:3089-3099. https://doi.org/10.1007/s11517-020-02261-2 
16. Casagrande A, Fabris F, Girometti R (2020) Extending information agreement by continuity. In: Proceedings - 2020 IEEE international conference on bioinformatics and biomedicine, BIBM 2020, pp 1432-1439. https://doi.org/10.1109/BIBM49941.2020.9313173

17. Chanda P, Costa E, Hu J, Sukumar S, Van Hemert J, Walia R (2020) Information theory in computational biology: where we stand today. Entropy 22(6):627

18. Chowdhary CL, Acharjya D (2020) Segmentation and feature extraction in medical imaging: a systematic review. Procedia Comput Sci 167:26-36

19. Cohen J (1960) A coefficient of agreement for nominal scales. Educ Psychol Meas 20:37-46. https://doi.org/10.1177/001316446002000104

20. Cover TM, Thomas JA (1991) Elements of information theory. Wiley series in telecommunications and signal processing. WileyInterscience, New York. https://doi.org/10.1002/0471200611

21. Cuesta-Frau D, Miro-Martinez P, Oltra-Crespo S, VarelaEntrecanales M, Aboy M, Novak D, Austin D (2009) Measuring body temperature time series regularity using approximate entropy and sample entropy. In: 2009 Annual International conference of the IEEE engineering in medicine and biology society. IEEE, pp 3461-3464

22. Delgado-Bonal A, Martín-Torres J (2016) Human vision is determined based on information theory. Sci Rep 6(1):1-5

23. Diamond GA (1991) Point of information. Med Decis Making 11:47-57

24. Diamond GA, Forrester JS, Hirsch M, Staniloff HM, Vas R, Berman DS, Swan HJ (1980) Application of conditional probability analysis to the clinical diagnosis of coronary artery disease. $\mathbf{J}$ Clin Investig 65:1210-1221. https://doi.org/10.1172/JCI109776

25. Diamond GA, Hirsch M, Forrester JS, Staniloff HM, Vas R, Halpern SW, Swan HJ (1981) Application of information theory to clinical diagnostic testing. The electrocardiographic stress test. Circulation 63:915-921. https://doi.org/10.1161/01.CIR.63.4.915

26. Dimitrov AG, Lazar AA, Victor JD (2011) Information theory in neuroscience. J Comput Neurosci 30(1):1-5

27. Fabris F (2009) Shannon information theory and molecular biology. J Interdiscip Math 12(1):41-87

28. Faes L, Nollo G, Porta A (2011) Information-based detection of nonlinear granger causality in multivariate processes via a nonuniform embedding technique. Phys Rev E 83(5):051,112

29. Faes L, Porta A (2014) Conditional entropy-based evaluation of information dynamics in physiological systems. In: Directed information measures in neuroscience. Springer, pp 61-86

30. Feixas M, Bardera A, Rigau J, Xu Q, Sbert M (2014) Information theory tools for image processing. Synth Lect Comput Graph Anim 6(1):1-164

31. Gehlbach S (1993) Interpretation: sensitivity, specificity, and predictive value. McGraw-Hill, New York, pp 129-139

32. Girometti R, Fabris F (2015) Informational analysis: a Shannon theoretic approach to measure the performance of a diagnostic test. Med Biol Eng Comput 53:899-910. https://doi.org/10.1007/s11517-015-1294-7

33. Girometti R, Zanotel M, Londero V, Bazzocchi M, Zuiani C (2017) Comparison between automated breast volume scanner (ABVS) versus hand-held ultrasound as a second look procedure after magnetic resonance imaging. Eur Radiol 27:3767-3775. https://doi.org/10.1007/s00330-017-4749-4

34. Giulini M, Menichetti R, Shell MS, Potestio R (2020) An information-theory-based approach for optimal model reduction of biomolecules. J Chem Theory Comput 16(11):6795-6813

35. Good IJ, Card WI (1971) The diagnostic process with special reference to errors. Methods Inform Med 10:176-188. https://doi.org/10.1055/s-0038-1636045
36. Granger CW (1969) Investigating causal relations by econometric models and cross-spectral methods. Econometrica: Journal of the Econometric Society, pp 424-438

37. Hu BG (2015) Information theory and its relation to machine learning. In: Proceedings of the 2015 Chinese Intelligent Automation Conference. Springer, pp 1-11

38. Jaynes ET (1957) Information theory and statistical mechanics. Phys Rev 106(4):620

39. Kang Y, Steis MR, Kolanowski AM, Fick D, Prabhu VV (2016) Measuring agreement between healthcare survey instruments using mutual information. BMC Med Inform Decis Mak, 16. https://doi.org/10.1186/s12911-016-0335-y

40. Kannan S, Kim H, Oh S (2018) Deep learning and information theory: an emerging interface. In: Tutorial at IEEE International symposium on information theory (ISIT)

41. Kessels SJ, Carter D, Ellery B, Newton S, Merlin TL (2020) Prenatal genetic testing for cystic fibrosis: a systematic review of clinical effectiveness and an ethics review. Genet Med 22(2):258 267

42. Khinchin AI (1958) Mathematical foundations of information theory. Dover Publications, New York. https://doi.org/10.2307/3610679

43. Klemens B (2012) Mutual information as a measure of intercoder agreement. J Off Stat 28(3):395-412. https://doi.org/10.5281/zenodo.3934825

44. Konopka AK (2003) Information theories in molecular biology and genomics

45. Kullback S, Leibler R (1951) On information and sufficiency. Ann Math Stat 22:79-86. https://doi.org/10.1214/aoms/1177729694

46. Lee WC (1999) Selecting diagnostic tests for ruling out or ruling in disease: the use of the Kullback-Leibler distance. Int $\mathrm{J}$ Epidemiol 28:521-525. https://doi.org/10.1093/ije/28.3.521

47. MacDonald D (1952) Information theory and its application to taxonomy. J Appl Phys 23(5):529-531

48. MacKay DJ, Mac Kay DJ (2003) Information theory, inference and learning algorithms. Cambridge University Press

49. Madani M, Nowroozi A (2011) Using information theory in pattern recognition for intrusion detection. J Theor Appl Inf Technol 34:138-142

50. Maes F, Vandermeulen D, Suetens P (2003) Medical image registration using mutual information. Proc IEEE 91(10):16991722

51. McHugh ML (2012) Interrater reliability: the kappa statistic. Biochem Med (Zagreb) 22(3):276-282

52. Metz CE, Goodenough DJ, Rossmann K (1973) Evaluation of receiver operating characteristic curve data in terms of information theory, with applications in radiography. Radiology 109:297-303. https://doi.org/10.1148/109.2.297

53. Montesinos I, Gruson D, Kabamba B, Dahma H, Van den Wijngaert S, Reza S, Carbone V, Vandenberg O, Gulbis B, Wolff F, Rodriguez-Villalobos H (2020) Evaluation of two automated and three rapid lateral flow immunoassays for the detection of anti-sars-cov-2 antibodies. J Clin Virol, 128. https://doi.org/10.1016/j.jcv.2020.104413

54. Montesinos L, Castaldo R, Pecchia L (2018) On the use of approximate entropy and sample entropy with centre of pressure time-series. J Neuroeng Rehab 15(1):1-15

55. Mossman D, Somoza E (1992) Diagnostic tests and information theory. J Neuropsychiatry Clin Neurosci 4(1):95-98

56. Okada M (1978) A method for clinical data reduction based on "weighted entropy". IEEE Trans Biomed Eng 25:462-467. https://doi.org/10.1109/TBME.1978.326352

57. Özlem EO, Armağan K (2011) Evaluation and comparison of diagnostic test performance based on information theory. Int J Stat Applic 1:10-13 
58. Peacock J, Peacock P (2010) Oxford handbook of medical statistics. Oxford University Press, Oxford. https://doi.org/10.1093/med/9780199551286.001.0001

59. Pisano ED, Gatsonis C, Hendrick E, Yaffe M, Baum JK, Acharyya S, Conant EF, Fajardo LL, Bassett L, D'Orsi C et al (2005) Diagnostic performance of digital versus film mammography for breast-cancer screening. England J Med 353(17):1773-1783

60. Porta A, Bari V, De Maria B, Cairo B, Vaini E, Malacarne M, Pagani M, Lucini D (2018) On the relevance of computing a local version of sample entropy in cardiovascular control analysis. IEEE Trans Biomed Eng 66(3):623-631

61. Porta A, De Maria B, Bari V, Marchi A, Faes L (2016) Are nonlinear model-free conditional entropy approaches for the assessment of cardiac control complexity superior to the linear model-based one? IEEE Trans Biomed Eng 64(6):1287-1296

62. Porta A, Faes L (2015) Wiener-Granger causality in network physiology with applications to cardiovascular control and neuroscience. Proc IEEE 104(2):282-309

63. Rao AR, Motiwala HG, Karim OM (2008) The discovery of prostate-specific antigen. BJU Int 101(1):5-10

64. Richman JS, Moorman JR (2000) Physiological time-series analysis using approximate entropy and sample entropy. Am J Physiol-Heart Circul Physiol 278(6):H2039-H2049

65. Rifkin RD (1985) Maximum Shannon information content of diagnostic medical testing: including application to multiple non-independent tests. Med Decis Making 5:179-190. https://doi.org/10.1177/0272989X8500500207

66. Ruiz FE, Pérez PS, Bonev BI (2009) Information theory in computer vision and pattern recognition. Springer Science \& Business Media

67. Shannon CE (1948) A mathematical theory of communication. Bell Syst Tech J 27:379-423. https://doi.org/10.1002/j.1538-7305.1948.tb01338.x

68. Shoukri MM (2004) Measures of interobserver agreement. CRC Biostatistics Series. Chapman \& Hall, Boca Raton. https://doi.org/10.1198/tech.2004.s205

69. Siggiridou E, Koutlis C, Tsimpiris A, Kugiumtzis D (2019) Evaluation of Granger causality measures for constructing networks from multivariate time series. Entropy 21(11):1080

70. Somoza E, Mossman D (1990) Optimizing rem latency as a diagnostic test for depression using receiver operating characteristic analysis and information theory. Biol Psych 27:990-1006. https://doi.org/10.1016/0006-3223(90)90036-2

71. Somoza E, Mossman D (1992) Comparing and optimizing diagnostic tests: an information-theoretical approach. Med Decis Making 12:179-188. https://doi.org/10.1177/0272989X9201200303

72. Somoza E, Mossman D (1992) Comparing diagnostic tests using information theory: The INFO-ROC technique. J Neuropsych Clin Neurosci 4:214-219. https://doi.org/10.1176/jnp.4.2.214

73. Somoza E, Soutullo-Esperon L, Mossman D (1989) Evaluation and optimization of diagnostic tests using receiver operating characteristic analysis and information theory. Int J Biomed Comput 24:153-189. https://doi.org/10.1016/0020-7101(89)90029-9

74. Uthoff J, Sieren JC (2018) Information theory optimization based feature selection in breast mammography lesion classification. In:
2018 IEEE 15th International symposium on biomedical imaging (ISBI 2018). IEEE, pp 817-821

75. Verdu S (1998) Fifty years of Shannon theory. IEEE Trans Inf Theory 44(6):2057-2078. https://doi.org/10.1109/18.720531

76. Warner J (2007) Linguistics and information theory: analytic advantages. J Am Soc Inf Sci Technol 58(2):275-285

77. Weinstein S, Obuchowski NA, Lieber ML (2005) Clinical evaluation of diagnostic tests. Am J Roentgenol 184:14-19. https://doi.org/10.2214/ajr.184.1.01840014

78. Wiener N (1956) The theory of prediction. Modern mathematics for engineers. NY, 165

79. Wu Y, Alagoz O, Ayvaci MU, Munoz Del Rio A, Vanness DJ, Woods R, Burnside ES (2013) A comprehensive methodology for determining the most informative mammographic features. J Digit Imag 26(5):941-947. https://doi.org/10.1007/s10278-013-9588-5

80. Xiong W, Faes L, Ivanov PC (2017) Entropy measures, entropy estimators, and their performance in quantifying complex dynamics: effects of artifacts, nonstationarity, and long-range correlations. Phys Rev E 95(6):062,114

81. Yang Y (2005) Information theory, inference and learning algorithms. J Am Stat Assoc 100(472):1461-1462. https://doi.org/ 10.1198/jasa.2005.s54

82. Zanelli E, Giannarini G, Cereser L, Zuiani C, Como G, Pizzolitto S, Crestani A, Valotto C, Ficarra V, Girometti R (2019) Head-tohead comparison between multiparametric MRI, the partin tables, memorial sloan kettering cancer center nomogram, and CAPRA score in predicting extraprostatic cancer in patients undergoing radical prostatectomy. J Magn Reson Imaging 50:1604-1613. https://doi.org/10.1002/jmri.26743

83. Zhou XH, Obuchowski NA, McClish DK (2011) Statistical methods in diagnostic medicine, 2nd edn. Wiley Series in Probability and Statistics. Wiley, New York. https://doi.org/10.1002/9780470906514

Publisher's note Springer Nature remains neutral with regard to jurisdictional claims in published maps and institutional affiliations.

Alberto Casagrande Assistant professor of Computer science, with main interests in the field of hybrid systems, formal verification, systems biology, and algorithms. Author of 30 international papers and proceedings.

Francesco Fabris Associate professor of Information processing systems, with main interests in the field of application of Shannon information theory and coding to medicine. Author of 40 international papers and proceedings

Rossano Girometti Associate professor of Radiology, focused on body MRI, and hepatic, pancreaticobiliary, gastrointestinal and prostate imaging. Author of more than 100 papers on international, peer-reviewed journals 